\title{
Research Article \\ Switching Signal Design for Global Exponential Stability of Uncertain Switched Neutral Systems
}

\author{
Ker-Wei Yu \\ Department of Marine Engineering, National Kaohsiung Marine University, Kaohsiung 811, Taiwan \\ Correspondence should be addressed to Ker-Wei Yu, kwyu@mail.nkmu.edu.tw
}

Received 22 March 2009; Revised 22 June 2009; Accepted 9 July 2009

Recommended by Tamas Kalmar-Nagy

The switching signal design for global exponential stability of switched neutral systems is investigated in this paper. LMI-based delay-dependent and delay-independent criteria are proposed to guarantee the global stability via the constructed switching signal. Razumikhin-like approach is used to find the stability results. Finally, some numerical examples are illustrated to show the main results.

Copyright (C) 2009 Ker-Wei Yu. This is an open access article distributed under the Creative Commons Attribution License, which permits unrestricted use, distribution, and reproduction in any medium, provided the original work is properly cited.

\section{Introduction}

It is well known that the existence of delay in a system may cause instability or bad system performance in control systems. Time-delay phenomenon appears in many practical systems, such as AIDS epidemic, aircraft stabilization, chemical engineering systems, inferred grinding model, manual control, neural network, nuclear reactor, population dynamic model, rolling mill, ship stabilization, and systems with lossless transmission lines. Hence stability analysis for time-delay systems has been considered in the recent years [1-3]. Neutral systems are described by functional differential equations which depend on the delays of state and state derivative. Some practical examples of neutral systems include distributed networks, heat exchanges, and processes including steam [4].

Switched system is a class of hybrid systems which is consisting of several subsystems and uses the switching signal to specify which subsystem is activated to the system trajectories at each instant of time. Some examples for switched systems are automated highway systems, constrained robotics, power systems and power electronics, transmission and stepper motors [5]. Stability analysis of switched time-delay systems has been an attractive research topic [6-13]. It is interesting to note that the stability for each subsystem cannot imply that of the overall system under arbitrary switching signal [9]. Another interesting fact is that the stability of a switched system can be achieved by choosing 
the switching signal even when each subsystem is unstable $[6,7,10]$. In this paper, the switching signal design will be considered for uncertain switched neutral systems with mixed delays. The switching signal will be proposed to guarantee the stability of switched system even when each subsystem is unstable. Based on Razumikhin-like approach [11], delaydependent and delay-independent results are provided. New and flexible LMI conditions are proposed to design the switching signal which guarantees the global exponential and asymptotic stability of uncertain switched neutral systems. Some numerical examples are provided to demonstrate the use of our results.

The notation used throughout this paper is as follows. For a matrix $A$, we denote the transpose by $A^{T}$, spectral norm by $\|A\|$, symmetric positive (negative) definite by $A>0(A<$ 0 ), maximal eigenvalue by $\lambda_{\max }(A)$, and minimal eigenvalue by $\lambda_{\min }(A) . A \leq B$ means that matrix $B-A$ is symmetric positive semidefinite. For two sets $X$ and $Y, X-Y$ means that the set of all points in $X$ that are not in $Y$. For a vector $x$, we denote the Euclidean norm by $\|x\|$

and $\left\|x_{t}\right\|_{s}=\sup _{-H \leq \theta \leq 0} \sqrt{\|x(t+\theta)\|^{2}+\|\dot{x}(t+\theta)\|^{2}}$. I denotes the identity matrix. $\mathfrak{R}^{n}$ denotes $n$-dimensional real space.

\section{Problem Formulations and Main Results}

Consider the following switched neutral system with mixed time delays:

$$
\begin{aligned}
\dot{x}(t)-D \dot{x}(t-\tau) & =A_{0 \sigma} x(t)+A_{1 \sigma} x(t-h(t)), \quad t \geq 0, \\
x(t) & =\phi(t), \quad t \in[-H, 0],
\end{aligned}
$$

where $x \in \Re^{n}, x_{t}$ is state at time $t$ defined by $x_{t}(\theta):=x(t+\theta), \forall \theta \in[-H, 0], \sigma$ is a switching signal which is a piecewise constant function and may depend on $t$ or $x, \sigma$, taking its values in the finite set $\{1,2, \ldots, N\}$, and time-varying delay satisfies $0 \leq h(t) \leq h_{M}, \dot{h}(t) \leq h_{D}, h_{M}>0$, $\tau>0, H=\max \left\{h_{M}, \tau\right\}$. Matrices $D, A_{0 i}$, and $A_{1 i} \in \mathfrak{R}^{n \times n}, i=1,2, \ldots, N$, are constant, and the initial vector $\phi \in C_{1}$, where $C_{1}$ is the set of differentiable functions from $[-H, 0]$ to $\mathfrak{R}^{n}$.

Now we define some functions $\lambda_{i}(t), i=1,2, \ldots, N$, that will be used to represent our system:

$$
\lambda_{i}(t)=\left\{\begin{array}{ll}
1, & \sigma=i, \\
0, & \text { otherwise, }
\end{array} \quad i=1,2, \ldots, N\right.
$$

The switched system in (2.1) can be rewritten as follows:

$$
\begin{gathered}
\dot{x}(t)-D \dot{x}(t-\tau)=\sum_{i=1}^{N} \lambda_{i}(t)\left\{A_{0 i} x(t)+A_{1 i} x(t-h(t))\right\}, \quad t \geq 0, \\
x(t)=\phi(t), \quad t \in[-H, 0],
\end{gathered}
$$

where $\lambda_{i}(t)$ is defined in (2.2) and $\sum_{i=1}^{N} \lambda_{i}(t)=1, \forall t \geq 0$. 
Lemma 2.1 (see [14]). Let $U, V, W$, and $M$ be real matrices of appropriate dimensions with $M$ satisfying $M=M^{T}$, then

$$
M+U V W+W^{T} V^{T} U^{T}<0 \quad \forall V^{T} V \leq I
$$

if and only if there exists a scalar $\varepsilon>0$ such that

$$
M+\varepsilon^{-1} \cdot U U^{T}+\varepsilon \cdot W^{T} W=M+\varepsilon^{-1} \cdot U U^{T}+\varepsilon^{-1} \cdot(\varepsilon W)^{T}(\varepsilon W)<0 .
$$

Lemma 2.2 (Schur complement of [15]). For a given matrix $S=\left[\begin{array}{ll}S_{11} & S_{12} \\ S_{12}^{T} & S_{22}\end{array}\right]$ with $S_{11}=S_{11}^{T}, S_{22}=$ $S_{22}^{T}$, the following conditions are equivalent:

(1) $S<0$,

(2) $S_{22}<0, S_{11}-S_{12} S_{22}^{-1} S_{12}^{T}<0$.

Assumption 2.3. Assume that there exists a convex combination $F=\sum_{i=1}^{N} \alpha_{i} A_{0 i}$ such that $F$ is Hurwitz, where $0 \leq \alpha_{i} \leq 1$ and $\sum_{i=1}^{N} \alpha_{i}=1$.

Since $F$ is Hurwitz, there exist positive definite matrices $P$ and $Q$ satisfying

$$
F^{T} P=P F \leq-Q
$$

Define some domains

$$
\Omega_{i}=\left\{x \in \mathfrak{R}^{n}: x^{T}\left(A_{0 i}^{T} P+P A_{0 i}\right) x \leq-x^{T} Q x\right\}, \quad i \in\{1,2, \ldots, N\} .
$$

From the similar proof of [7], it is easy to show $\bigcup_{i=1}^{N} \Omega_{i}=\mathfrak{R}^{n}$. Construct some domains

$$
\bar{\Omega}_{1}=\Omega_{1}, \bar{\Omega}_{2}=\Omega_{2}-\bar{\Omega}_{1}, \bar{\Omega}_{3}=\Omega_{3}-\bar{\Omega}_{1}-\bar{\Omega}_{2}, \ldots, \bar{\Omega}_{N}=\Omega_{N}-\bar{\Omega}_{1}-\cdots-\bar{\Omega}_{N-1} .
$$

We can obtain $\bigcup_{i=1}^{N} \bar{\Omega}_{i}=\Re^{n}$ and $\bar{\Omega}_{i} \cap \bar{\Omega}_{j}=\Phi, i \neq j$, where $\Phi$ is an empty set. If Assumption 2.3 is satisfied, then the following results can be derived:

$$
x^{T}\left(A_{0 i}^{T} P+P A_{0 i}\right) x \leq-x^{T} Q x, \quad x(t) \in \bar{\Omega}_{i} .
$$

Define the following switching function:

$$
\sigma=i, \quad \forall x \in \bar{\Omega}_{i}
$$

Definition 2.4 (see [14]). The system (2.1) with the designed switching signal is said to be the globally exponentially stabilizable with convergence rate $\alpha>0$ by the designed switching signal, if there are two positive constants $\alpha$ and $\Psi$ such that

$$
\|x(t)\| \leq \Psi \cdot e^{-\alpha t}, \quad t \geq 0 .
$$


Now we present a result to design the switching signal that guarantees global exponential stability of system (2.1).

Theorem 2.5. Assume that for $\|D\|<1,0<\alpha<-(\ln \|D\|) / \tau, 0 \leq \alpha_{i} \leq 1, i=1,2, \ldots, N$, and $\sum_{i=1}^{N} \alpha_{i}=1$, there exist some $n \times n$ matrices $P, Q, R_{1}, R_{2}>0$, such that the following LMI conditions hold for all $i=1,2, \ldots, N$ :

$$
\begin{gathered}
\Xi_{i}=\left[\begin{array}{ccc}
\Xi_{11 i} & \Xi_{12 i} & \Xi_{13 i} \\
* & \Xi_{22 i} & \Xi_{23 i} \\
* & * & \Xi_{33 i}
\end{array}\right]<0, \\
F^{T} P+P F+Q<0, \quad F=\sum_{i=1}^{N} \alpha_{i} A_{0 i},
\end{gathered}
$$

where

$$
\begin{gathered}
\Xi_{11 i}=2 \alpha \cdot P+R_{1}+R_{2}-Q, \quad \Xi_{12 i}=-2 \alpha \cdot P D-A_{0 i}{ }^{T} P D, \quad \Xi_{13 i}=P A_{1 i}, \\
\Xi_{22 i}=2 \alpha \cdot D^{T} P D-e^{-2 \alpha \tau} \cdot R_{1}, \quad \Xi_{23 i}=-D^{T} P A_{1 i}, \quad \Xi_{33 i}=-\left(1-h_{D}\right) \cdot e^{-2 \alpha h_{M}} \cdot R_{2} .
\end{gathered}
$$

Then the system (2.1) is globally exponentially stabilizable with convergence rate $\alpha$ by the switching signal given in (2.10).

Proof. Define the Lyapunov functional

$$
\begin{aligned}
V\left(x_{t}\right)= & e^{2 \alpha t} \cdot(x(t)-D x(t-\tau))^{T} P(x(t)-D x(t-\tau)) \\
& +\int_{t-\tau}^{t} e^{2 \alpha s} \cdot x^{T}(s) R_{1} x(s) d s+\int_{t-h(t)}^{t} e^{2 \alpha s} \cdot x^{T}(s) R_{2} x(s) d s,
\end{aligned}
$$

where $P, R_{1}, R_{2}>0$. The time derivatives of $V\left(x_{t}\right)$ along the trajectories of system (2.3) under the switching function (2.10) satisfy

$$
\begin{aligned}
\dot{V}\left(x_{t}\right)= & e^{2 \alpha t} \cdot\left[2 \alpha \cdot(x(t)-D x(t-\tau))^{T} P(x(t)-D x(t-\tau))\right] \\
& +e^{2 \alpha t} \cdot \sum_{i=1}^{N} \lambda_{i}(t)\left[\left(A_{0 i} x(t)+A_{1 i} x(t-h(t))\right)^{T} P(x(t)-D x(t-\tau))\right] \\
& +e^{2 \alpha t} \cdot \sum_{i=1}^{N} \lambda_{i}(t)\left[(x(t)-D x(t-\tau))^{T} P\left(A_{0 i} x(t)+A_{1 i} x(t-h(t))\right)\right] \\
& +e^{2 \alpha t} \cdot\left[x^{T}(t) R_{1} x(t)-e^{-2 \alpha \tau} \cdot x^{T}(t-\tau) R_{1} x(t-\tau)\right] \\
& +e^{2 \alpha t} \cdot\left[x^{T}(t) R_{2} x(t)-(1-\dot{h}(t)) \cdot e^{-2 \alpha h(t)} \cdot x^{T}(t-h(t)) R_{2} x(t-h(t))\right] .
\end{aligned}
$$


By the condition (2.9) and switching function (2.10), we obtain

$$
\dot{V}\left(x_{t}\right) \leq e^{2 \alpha t} \cdot \sum_{i=1}^{N} \lambda_{i}(t) \cdot\left[X^{T} \cdot \Xi_{i} \cdot X\right]
$$

where $\Xi_{i}, i=1,2, \ldots, N$, are defined in (2.12), $X^{T}=\left[\begin{array}{lll}x^{T}(t) & x^{T}(t-\tau) & x^{T}(t-h(t))\end{array}\right]$. From (2.16) with $\Xi_{i}<0$, we have

$$
V\left(x_{t}\right) \leq V\left(x_{0}\right), \quad t \geq 0,
$$

where

$$
V\left(x_{0}\right) \leq \delta_{1} \cdot\left\|x_{0}\right\|_{s^{\prime}}^{2} \quad \delta_{1}=\lambda_{\max }(P)(1+\|D\|)^{2}+\tau \cdot \lambda_{\max }\left(R_{1}\right)+h_{M} \cdot \lambda_{\max }\left(R_{2}\right) .
$$

From (2.14), we have

$$
\lambda_{\min }(P) \cdot e^{2 \alpha t} \cdot\|\wp(t)\|^{2} \leq e^{2 \alpha t} \cdot \wp^{T}(t) P \wp(t) \leq V\left(x_{t}\right) \leq \delta_{1} \cdot\left\|x_{0}\right\|_{S^{\prime}}^{2}
$$

where $\wp(t)=x(t)-D x(t-\tau)$. From (2.19), we can obtain

$$
\|x(t)\|=\|\wp(t)+D x(t-\tau)\| \leq\|D\| \cdot\|x(t-\tau)\|+\|\wp(t)\| \leq\|D\| \cdot\|x(t-\tau)\|+\delta_{2} \cdot e^{-\alpha t}, \quad t \geq 0,
$$

where $\delta_{2}=\sqrt{\delta_{1} / \lambda_{\min }(P)} \cdot\left\|x_{0}\right\|_{s}$. Since $\|D\|<1$ and $\tau>0$, we can choose a sufficiently small positive constant $\xi=\alpha<-(\ln \|D\|) / \tau$ satisfying $\|D\| \cdot e^{\xi \tau}<1$. By the Razumikhin-like approach of [14], we have

$$
\|x(t)\| \leq\left[\left\|x_{0}\right\|_{s}+\frac{\delta_{2}}{1-\|D\| e^{\xi h}}\right] \cdot e^{-\xi t}, \quad t \geq 0
$$

This completes the proof.

Consider the following uncertain switched neutral system with mixed time delays:

$$
\begin{gathered}
\dot{x}(t)-D \dot{x}(t-\tau)=\left[A_{0 \sigma}+\Delta A_{0 \sigma}(t)\right] x(t)+\left[A_{1 \sigma}+\Delta A_{1 \sigma}(t)\right] x(t-h(t)), \quad t \geq 0, \\
x(t)=\phi(t), \quad t \in[-H, 0],
\end{gathered}
$$

where $\Delta A_{0 i}(t)$ and $\Delta A_{1 i}(t)$ are some perturbed matrices and satisfy the following condition:

$$
\left[\Delta A_{0 i}(t) \quad \Delta A_{1 i}(t)\right]=M_{i} F_{i}(t)\left[N_{0 i} N_{1 i}\right], \quad \forall i \in\{1,2, \ldots, N\}, t \geq 0,
$$


where $M_{i}, N_{0 i}$, and $N_{1 i}, i=1,2, \ldots, N$, are some given constant matrices with appropriate dimensions, and $F_{i}(t), i=1,2, \ldots, N$, are unknown matrices representing the parameter perturbation which satisfy

$$
F_{i}^{T}(t) F_{i}(t) \leq I, \quad \forall i \in\{1,2, \ldots, N\}, t \geq 0
$$

The uncertain switched system in (2.22a)-(2.22c) can be rewritten as follows:

$$
\begin{gathered}
\dot{x}(t)-D \dot{x}(t-\tau)=\sum_{i=1}^{N} \lambda_{i}(t)\left\{\left[A_{0 i}+\Delta A_{0 i}(t)\right] x(t)+\left[A_{1 i}+\Delta A_{1 i}(t)\right] x(t-h(t))\right\}, \quad t \geq 0, \\
x(t)=\phi(t), \quad t \in[-H, 0],
\end{gathered}
$$

where $\lambda_{i}(t)$ is defined in (2.2) and $\sum_{i=1}^{N} \lambda_{i}(t)=1, \forall t \geq 0$. (2.22c).

Now we consider the exponential stability for uncertain switched system (2.22a)-

Theorem 2.6. Assume that for $\|D\|<1,0<\alpha<-(\ln \|D\|) / \tau, 0 \leq \alpha_{i} \leq 1, i=1,2, \ldots, N$, and $\sum_{i=1}^{N} \alpha_{i}=1$, there exist constants $\varepsilon_{i}>0, i=1,2, \ldots, N$, and some $n \times n$ matrices $P, Q, R_{1}, R_{2}>0$, such that the following LMI conditions hold for all $i=1,2, \ldots, N$ :

$$
\begin{gathered}
\bar{\Xi}_{i}=\left[\begin{array}{ccccc}
\Xi_{11 i} & \Xi_{12 i} & \Xi_{13 i} & \bar{\Xi}_{14 i} & \bar{\Xi}_{15 i} \\
* & \Xi_{22 i} & \Xi_{23 i} & \bar{\Xi}_{24 i} & 0 \\
* & * & \Xi_{33 i} & 0 & \bar{\Xi}_{35 i} \\
* & * & * & \bar{\Xi}_{44 i} & 0 \\
* & * & * & * & \bar{\Xi}_{55 i}
\end{array}\right]<0, \\
F^{T} P+P F+Q<0, \quad F=\sum_{i=1}^{N} \alpha_{i} A_{0 i}
\end{gathered}
$$

where $\Xi_{j k i}, j, k=1,2,3$, are defined in (2.12)

$$
\bar{\Xi}_{14 i}=P M_{i}, \quad \bar{\Xi}_{15 i}=\varepsilon_{i} \cdot N_{0 i}^{T}, \quad \bar{\Xi}_{24 i}=-D^{T} P M_{i}, \quad \bar{\Xi}_{35 i}=\varepsilon_{i} \cdot N_{1 i}^{T}, \quad \bar{\Xi}_{44 i}=\bar{\Xi}_{55 i}=-\varepsilon_{i} \cdot I .
$$

Then the system (2.22a)-(2.22c) is globally exponentially stabilizable with convergence rate $\alpha$ by the switching signal given in (2.10).

Proof. The time derivatives of $V\left(x_{t}\right)$ in (2.14) along the trajectories of system (2.22a)-(2.22c) under the switching function (2.9) satisfy

$$
\dot{V}\left(x_{t}\right) \leq e^{2 \alpha t} \cdot \sum_{i=1}^{N} \lambda_{i}(t) \cdot\left[X^{T} \cdot \widehat{\Xi}_{i} \cdot X\right],
$$


where

$$
\widehat{\Xi}_{i}=\Xi_{i}+\left[\begin{array}{c}
P M_{i} \\
-D^{T} P M_{i} \\
0
\end{array}\right] F_{i}(t)\left[\begin{array}{lll}
N_{0 i} & 0 & N_{1 i}
\end{array}\right]+\left[\begin{array}{c}
N_{0 i}^{T} \\
0 \\
N_{1 i}^{T}
\end{array}\right] F_{i}^{T}(t)\left[\begin{array}{lll}
M_{i}^{T} P & -M_{i}^{T} P D & 0
\end{array}\right] .
$$

By Lemmas 2.1 and 2.2, the condition $\bar{\Xi}_{i}<0$ in (2.25) is equivalent to $\widehat{\Xi}_{i}<0$. By the same derivation of Theorem 2.5 , this proof can be completed.

If we choose the convergence rate $\alpha=0$, we can obtain the following delayindependent condition for the global asymptotic stability of system (2.22a) $-(2.22 \mathrm{c})$.

Corollary 2.7. Assume that for $\|D\|<1,0 \leq \alpha_{i} \leq 1, i=1,2, \ldots, N$, and $\sum_{i=1}^{N} \alpha_{i}=1$, there exist constants $\varepsilon_{i}>0, i=1,2, \ldots, N$, some $n \times n$ matrices $P, Q, R_{1}, R_{2}>0$, such that the following $L M I$ conditions hold for all $i=1,2, \ldots, N$ :

$$
\begin{aligned}
& \widetilde{\Xi}_{i}=\left[\begin{array}{ccccc}
\widetilde{\Xi}_{11 i} & \widetilde{\Xi}_{12 i} & \widetilde{\Xi}_{13 i} & \widetilde{\Xi}_{14 i} & \widetilde{\Xi}_{15 i} \\
* & \widetilde{\Xi}_{22 i} & \widetilde{\Xi}_{23 i} & \widetilde{\Xi}_{24 i} & 0 \\
* & * & \widetilde{\Xi}_{33 i} & 0 & \widetilde{\Xi}_{35 i} \\
* & * & * & \widetilde{\Xi}_{44 i} & 0 \\
* & * & * & * & \widetilde{\Xi}_{55 i}
\end{array}\right]<0, \\
& F^{T} P+P F+Q<0, \quad F=\sum_{i=1}^{N} \alpha_{i} A_{0 i},
\end{aligned}
$$

where

$$
\begin{gathered}
\widetilde{\Xi}_{11 i}=R_{1}+R_{2}-Q, \quad \tilde{\Xi}_{12 i}=-A_{0 i}^{T} P D, \quad \tilde{\Xi}_{13 i}=P A_{1 i}, \quad \tilde{\Xi}_{14 i}=P M_{i}, \quad \tilde{\Xi}_{15 i}=\varepsilon_{i} \cdot N_{0 i}^{T}, \\
\widetilde{\Xi}_{22 i}=-R_{1}, \quad \widetilde{\Xi}_{23 i}=-D^{T} P A_{1 i}, \quad \widetilde{\Xi}_{24 i}=-D^{T} P M_{i}, \quad \widetilde{\Xi}_{33 i}=-\left(1-h_{D}\right) \cdot R_{2}, \\
\widetilde{\Xi}_{35 i}=\varepsilon_{i} \cdot N_{1 i}^{T}, \quad \tilde{\Xi}_{44 i}=\widetilde{\Xi}_{55 i}=-\varepsilon_{i} \cdot I .
\end{gathered}
$$

Then the system (2.22a)-(2.22c) is globally asymptotically stabilizable by the switching signal given in (2.10). 
If $D=0$, Corollary 2.7 can be reduced to the following corollary.

Corollary 2.8. Assume that for some constants $\alpha_{i}, i=1,2, \ldots, N$, there exist constants $\varepsilon_{i}>0$, $i=1,2, \ldots, N$, some $n \times n$ matrices $P, Q, R_{2}>0$, such that the following $L M I$ conditions hold for all $i=1,2, \ldots, N$ :

$$
\begin{gathered}
\widetilde{\Xi}_{i}=\left[\begin{array}{cccc}
\widetilde{\Xi}_{11 i} & \widetilde{\Xi}_{12 i} & \widetilde{\Xi}_{13 i} & \widetilde{\Xi}_{14 i} \\
* & \widetilde{\Xi}_{22 i} & 0 & \widetilde{\Xi}_{24 i} \\
* & * & \widetilde{\Xi}_{33 i} & 0 \\
* & * & * & \widetilde{\Xi}_{44 i}
\end{array}\right]<0, \\
F^{T} P+P F+Q<0, \quad F=\sum_{i=1}^{N} \alpha_{i} A_{0 i},
\end{gathered}
$$

where

$$
\begin{aligned}
& \tilde{\widetilde{\Xi}}_{11 i}=R_{2}-Q, \quad \tilde{\widetilde{\Xi}}_{12 i}=P A_{1 i}, \quad \tilde{\widetilde{\Xi}}_{13 i}=P M_{i}, \quad \widetilde{\widetilde{\Xi}}_{14 i}=\varepsilon_{i} \cdot N_{0 i}^{T}, \quad \widetilde{\widetilde{\Xi}}_{22 i}=-\left(1-h_{D}\right) \cdot R_{2}, \\
& \widetilde{\Xi}_{24 i}=\varepsilon_{i} \cdot N_{1 i}^{T}, \quad \widetilde{\Xi}_{33 i}=\widetilde{\Xi}_{44 i}=-\varepsilon_{i} \cdot I .
\end{aligned}
$$

Then the system (2.22a)-(2.22c) is globally asymptotically stabilizable by the switching signal given in (2.10).

Assumption 2.9. Assume that there exists a convex combination $F=\sum_{i=1}^{N} \alpha_{i} A_{0 i}$, some positive definite matrices $P$ and $Q$, some matrices $\widehat{S}_{i}, i=1,2, \ldots, N$, such that

$$
F^{T} P+P F+\sum_{i=1}^{N} \alpha_{i} \cdot\left(\widehat{S}_{i}+\widehat{S}_{i}^{T}\right)<-Q
$$

where $0 \leq \alpha_{i} \leq 1$ and $\sum_{i=1}^{N} \alpha_{i}=1$.

Define some domains

$$
\widehat{\Omega}_{i}=\left\{x \in \Re^{n}: x^{T}\left(A_{0 i}{ }^{T} P+P A_{0 i}+\widehat{S}_{i}+\widehat{S}_{i}^{T}\right) x \leq-x^{T} Q x\right\}, \quad i \in\{1,2, \ldots, N\} .
$$

From the similar proof of [7], it is easy to show $\bigcup_{i=1}^{N} \widehat{\Omega}_{i}=\mathfrak{R}^{n}$. Construct some domains

$$
\widetilde{\Omega}_{1}=\widehat{\Omega}_{1}, \widetilde{\Omega}_{2}=\widehat{\Omega}_{2}-\widetilde{\Omega}_{1}, \widetilde{\Omega}_{3}=\widehat{\Omega}_{3}-\widetilde{\Omega}_{1}-\widetilde{\Omega}_{2}, \ldots, \widetilde{\Omega}_{N}=\widehat{\Omega}_{N}-\widetilde{\Omega}_{1}-\cdots-\widetilde{\Omega}_{N-1}
$$


We can obtain $\bigcup_{i=1}^{N} \widetilde{\Omega}_{i}=\Re^{n}$ and $\widetilde{\Omega}_{i} \cap \widetilde{\Omega}_{j}=\Phi, i \neq j$, where $\Phi$ is an empty set. If Assumption 2.9 is satisfied, then the following results can be derived:

$$
x^{T}\left(A_{0 i}^{T} P+P A_{0 i}+\widehat{S}_{i}+\widehat{S}_{i}^{T}\right) x \leq-x^{T} Q x, \quad x(t) \in \tilde{\Omega}_{i} .
$$

Define the following switching function:

$$
\sigma=i, \quad \forall x \in \widetilde{\Omega}_{i}
$$

Remark 2.10. In $[6,7,10]$, their assumption is given by

$$
F_{1}^{T} P+P F_{1}=-Q
$$

where $F_{1}=\sum_{i=1}^{N} \alpha_{i} \cdot\left(A_{0 i}+A_{1 i}\right)$. We can see that our Assumption 2.9 is more flexible with $\widehat{S}_{i}=P A_{1 i}, \forall i=1,2, \ldots, N$. The main difference of Assumptions 2.3 and 2.9 is that some matrices $\widehat{S}_{i}$ are introduced in Assumption 2.9. These matrices play a key role to derive the delay-dependent results.

Theorem 2.11. Assume that for $\|D\|<1,0<\alpha<-(\ln \|D\|) / \tau, 0 \leq \alpha_{i} \leq 1, i=1,2, \ldots, N$, and $\sum_{i=1}^{N} \alpha_{i}=1$, there exist constants $\varepsilon_{i}>0, i=1,2, \ldots, N$, some $n \times n$ matrices $P, Q, R_{1}, R_{2}, R_{3}, R_{4}>0$, and some $n \times n$ matrices $R_{12}, U, \widehat{S}_{i}, V_{1 i}, V_{2 i}$, and $V_{3 i}, i=1,2, \ldots, N$, such that the following LMI conditions hold for all $i=1,2, \ldots, N$ :

$$
\begin{aligned}
& \bar{\Sigma}_{i}=\left[\begin{array}{cccccccc}
\Sigma_{11 i} & \Sigma_{12 i} & \Sigma_{13 i} & \Sigma_{14 i} & \Sigma_{15 i} & \Sigma_{16 i} & \Sigma_{17 i} & \Sigma_{18 i} \\
* & \Sigma_{22 i} & 0 & \Sigma_{24 i} & \Sigma_{25 i} & 0 & \Sigma_{27 i} & 0 \\
* & * & \Sigma_{33 i} & \Sigma_{34 i} & \Sigma_{35 i} & \Sigma_{36 i} & \Sigma_{37 i} & 0 \\
* & * & * & \Sigma_{44 i} & \Sigma_{45 i} & 0 & \Sigma_{47 i} & 0 \\
* & * & * & * & \Sigma_{55 i} & \Sigma_{56 i} & 0 & \Sigma_{58 i} \\
* & * & * & * & * & \Sigma_{66 i} & 0 & 0 \\
* & * & * & * & * & * & \Sigma_{77 i} & 0 \\
* & * & * & * & * & * & * & \Sigma_{88 i}
\end{array}\right]<0, \\
& F^{T} P+P F+\sum_{i=1}^{N} \alpha_{i} \cdot\left(\widehat{S}_{i}+\widehat{S}_{i}^{T}\right)+Q<0, \quad F=\sum_{i=1}^{N} \alpha_{i} A_{0 i}, \\
& {\left[\begin{array}{cc}
R_{1} & R_{12} \\
* & R_{2}
\end{array}\right]>0}
\end{aligned}
$$


where

$$
\begin{gathered}
\Sigma_{11 i}=2 \alpha \cdot P-Q+R_{1}+R_{3}-V_{1 i}-V_{1 i}^{T}, \quad \Sigma_{12 i}=R_{12}+A_{0 i}^{T} U^{T}, \\
\Sigma_{13 i}=-2 \alpha P D-A_{0 i}{ }^{T} P D-\widehat{S}_{i}^{T} D, \quad \Sigma_{14 i}=-A_{0 i}{ }^{T} U^{T} D, \\
\Sigma_{15 i}=P A_{1 i}-\widehat{S}_{i}-V_{2 i}+V_{1 i}^{T}, \quad \Sigma_{16 i}=-\widehat{S}_{i}-V_{3 i}+V_{1 i}^{T}, \quad \Sigma_{17 i}=P M_{i}, \quad \Sigma_{18 i}=\varepsilon_{i} \cdot N_{0 i}^{T}, \\
\Sigma_{22 i}=R_{2}+h_{M}^{2} \cdot R_{4}-U-U^{T}, \quad \Sigma_{24 i}=\left(U+U^{T}\right) D, \quad \Sigma_{25 i}=U A_{1 i}, \quad \Sigma_{27 i}=U M_{i}, \\
\Sigma_{33 i}=2 \alpha D^{T} P D-e^{-2 \alpha \tau} \cdot R_{1}, \quad \Sigma_{34 i}=-e^{-2 \alpha \tau} \cdot R_{12}, \quad \Sigma_{35 i}=-D^{T} P A_{1 i}+D^{T} \widehat{S}_{i}, \quad \Sigma_{36 i}=D^{T} \widehat{S}_{i}, \\
\Sigma_{37 i}=-D^{T} P M_{i}, \quad \Sigma_{44 i}=-e^{-2 \alpha \tau} \cdot R_{2}-D^{T}\left(U+U^{T}\right) D, \quad \Sigma_{45 i}=-D^{T} U A_{1 i}, \quad \Sigma_{47 i}=-D^{T} U M_{i}, \\
\Sigma_{55 i}=-\left(1-h_{D}\right) \cdot e^{-2 \alpha h_{M}} \cdot R_{3}+V_{2 i}+V_{2 i}^{T}, \quad \Sigma_{56 i}=V_{3 i}+V_{2 i}^{T}, \quad \Sigma_{58 i}=\varepsilon_{i} \cdot N_{1 i}^{T}, \\
\Sigma_{66 i}=-e^{-2 \alpha h_{M}} \cdot R_{4}+V_{3 i}+V_{3 i}^{T}, \quad \Sigma_{77 i}=\Sigma_{88 i}=-\varepsilon_{i} \cdot I .
\end{gathered}
$$

Then the system (2.22a)-(2.22c) is globally exponentially stabilizable with convergence rate $\alpha$ by the switching signal given in (2.37).

Proof. Define the Lyapunov functional

$$
\begin{aligned}
V\left(x_{t}\right)= & e^{2 \alpha t} \cdot(x(t)-D x(t-\tau))^{T} P(x(t)-D x(t-\tau)) \\
& +\int_{t-\tau}^{t} e^{2 \alpha s} \cdot\left[x^{T}(s) \dot{x}^{T}(s)\right]\left[\begin{array}{cc}
R_{1} & R_{12} \\
* & R_{2}
\end{array}\right]\left[\begin{array}{c}
x(s) \\
\dot{x}(s)
\end{array}\right] d s \\
& +\int_{t-h(t)}^{t} e^{2 \alpha s} \cdot x^{T}(s) R_{3} x(s) d s+h_{M} \cdot \int_{t-h_{M}}^{t} e^{2 \alpha s} \cdot\left(s-\left(t-h_{M}\right)\right) \dot{x}^{T}(s) R_{4} \dot{x}(s) d s,
\end{aligned}
$$

where $P, R_{3}, R_{4}>0,\left[\begin{array}{cc}R_{1} & R_{12} \\ * & R_{2}\end{array}\right]>0$. The time derivatives of $V\left(x_{t}\right)$ along the trajectories of system (2.24) satisfy

$$
\begin{aligned}
\dot{V}\left(x_{t}\right)=e^{2 \alpha t} \cdot\left[2 \alpha \cdot(x(t)-D x(t-\tau))^{T} P(x(t)-D x(t-\tau))\right] & \\
+e^{2 \alpha t} \cdot \sum_{i=1}^{N} \lambda_{i}(t) & {\left[\left(\left(A_{0 i}+S_{i}+\Delta A_{0 i}(t)\right) x(t)-S_{i} \cdot \int_{t-h(t)}^{t} \dot{x}(s) d s\right.\right.} \\
& \left.\left.+\left(A_{1 i}+\Delta A_{1 i}(t)-S_{i}\right) x(t-h(t))\right)^{T} P(x(t)-D x(t-\tau))\right] \\
+e^{2 \alpha t} \cdot \sum_{i=1}^{N} \lambda_{i}(t) & {\left[( x ( t ) - D x ( t - \tau ) ) ^ { T } P \left(\left(A_{0 i}+S_{i}+\Delta A_{0 i}(t)\right) x(t)-S_{i} \cdot \int_{t-h(t)}^{t} \dot{x}(s) d s\right.\right.} \\
& \left.\left.+\left(A_{1 i}+\Delta A_{1 i}(t)-S_{i}\right) x(t-h(t))\right)\right]
\end{aligned}
$$


Mathematical Problems in Engineering

$$
\begin{aligned}
&+e^{2 \alpha t} \cdot[ {\left[x^{T}(t) \dot{x}^{T}(t)\right]\left[\begin{array}{cc}
R_{1} & R_{12} \\
* & R_{2}
\end{array}\right]\left[\begin{array}{c}
x(t) \\
\dot{x}(t)
\end{array}\right] } \\
&\left.-e^{-2 \alpha \tau} \cdot\left[x^{T}(t-\tau) \dot{x}^{T}(t-\tau)\right]\left[\begin{array}{cc}
R_{1} & R_{12} \\
* & R_{2}
\end{array}\right]\left[\begin{array}{c}
x(t-\tau) \\
\dot{x}(t-\tau)
\end{array}\right]\right] \\
&+e^{2 \alpha t} \cdot\left[x^{T}(t) R_{3} x(t)-(1-\dot{h}(t)) \cdot e^{-2 \alpha h(t)} \cdot x^{T}(t-h(t)) R_{3} x(t-h(t))\right] \\
&+e^{2 \alpha t} \cdot\left[h_{M}^{2} \cdot \dot{x}^{T}(t) R_{4} \dot{x}(t)-h_{M} \cdot \int_{t-h_{M}}^{t} e^{2 \alpha(s-t)} \cdot \dot{x}^{T}(s) R_{4} \dot{x}(s) d s\right],
\end{aligned}
$$

where $\widehat{S}_{i}=P S_{i}$. By the inequality in [1, page 322], we have

$$
\begin{aligned}
-h_{M} \cdot \int_{t-h_{M}}^{t} e^{2 \alpha s} \cdot \dot{x}^{T}(s) R_{4} \dot{x}(s) d s & \leq-h(t) \cdot e^{2 \alpha t} \cdot e^{-2 \alpha h_{M}} \cdot \int_{t-h(t)}^{t} \dot{x}^{T}(s) R_{4} \dot{x}(s) d s \\
& \leq-e^{2 \alpha t} \cdot e^{-2 \alpha h_{M}} \cdot\left[\int_{t-h(t)}^{t} \dot{x}(s) d s\right]^{T} R_{4}\left[\int_{t-h(t)}^{t} \dot{x}(s) d s\right] .
\end{aligned}
$$

By system (2.24) and Leibniz-Newton formula, we have

$$
\begin{aligned}
& \sum_{i=1}^{N} \lambda_{i}(t) \cdot e^{2 \alpha t} \cdot\left[\int_{t-h(t)}^{t} \dot{x}(s) d s-x(t)+x(t-h(t))\right]^{T}\left[V_{1 i} x(t)+V_{2 i} x(t-h(t))+V_{3 i} \int_{t-h(t)}^{t} \dot{x}(s) d s\right] \\
& \quad+\sum_{i=1}^{N} \lambda_{i}(t) \cdot e^{2 \alpha t} \cdot\left[V_{1 i} x(t)+V_{2 i} x(t-h(t))+V_{3 i} \int_{t-h(t)}^{t} \dot{x}(s) d s\right]^{T} \\
& \quad \times\left[\int_{t-h(t)}^{t} \dot{x}(s) d s-x(t)+x(t-h(t))\right]=0, \\
& -e^{2 \alpha t} \cdot(\dot{x}(t)-D \dot{x}(t-\tau))^{T}\left(U+U^{T}\right)(\dot{x}(t)-D \dot{x}(t-\tau)) \\
& \quad+\sum_{i=1}^{N} \lambda_{i}(t) \cdot e^{2 \alpha t} \cdot(\dot{x}(t)-D \dot{x}(t-\tau))^{T} U\left\{\left[A_{0 i}+\Delta A_{0 i}(t)\right] x(t)+\left[A_{1 i}+\Delta A_{1 i}(t)\right] x(t-h(t))\right\} \\
& \quad+\sum_{i=1}^{N} \lambda_{i}(t) \cdot e^{2 \alpha t} \cdot\left\{\left[A_{0 i}+\Delta A_{0 i}(t)\right] x(t)+\left[A_{1 i}+\Delta A_{1 i}(t)\right] x(t-h(t))\right\}^{T} U^{T}(\dot{x}(t)-D \dot{x}(t-\tau))=0 .
\end{aligned}
$$

By the conditions (2.42)-(2.44), we obtain the following result:

$$
\dot{V}\left(x_{t}\right) \leq e^{2 \alpha t} \cdot \sum_{i=1}^{N} \lambda_{i}(t) \cdot\left[X^{T} \cdot \Sigma_{i} \cdot X\right]
$$


where

$$
\begin{aligned}
& X^{T}=\left[\begin{array}{llllll}
x^{T}(t) & \dot{x}^{T}(t) & x^{T}(t-\tau) & \dot{x}^{T}(t-\tau) & x^{T}(t-h(t)) & \int_{t-h(t)}^{t} \dot{x}^{T}(s) d s
\end{array}\right], \\
& \Sigma_{i}=\left[\begin{array}{cccccc}
\Sigma_{11 i} & \Sigma_{12 i} & \Sigma_{13 i} & \Sigma_{14 i} & \Sigma_{15 i} & \Sigma_{16 i} \\
* & \Sigma_{22 i} & 0 & \Sigma_{24 i} & \Sigma_{25 i} & 0 \\
* & * & \Sigma_{33 i} & \Sigma_{34 i} & \Sigma_{35 i} & \Sigma_{36 i} \\
* & * & * & \Sigma_{44 i} & \Sigma_{45 i} & 0 \\
* & * & * & * & \Sigma_{55 i} & \Sigma_{56 i} \\
* & * & * & * & * & \Sigma_{66 i}
\end{array}\right]+\left[\begin{array}{c}
P M_{i} \\
U M_{i} \\
-D^{T} P M_{i} \\
-D^{T} U M_{i} \\
0 \\
0
\end{array}\right] F_{i}(t)\left[\begin{array}{c}
N_{0 i}^{T} \\
0 \\
0 \\
0 \\
N_{1 i}^{T} \\
0
\end{array}\right]^{T} \\
& +\left[\begin{array}{c}
N_{0 i}^{T} \\
0 \\
0 \\
0 \\
N_{1 i}^{T} \\
0
\end{array}\right] F_{i}^{T}(t)\left[\begin{array}{c}
P M_{i} \\
U M_{i} \\
-D^{T} P M_{i} \\
-D^{T} U M_{i} \\
0 \\
0
\end{array}\right]
\end{aligned}
$$

By Lemmas 2.1 and 2.2, the condition $\bar{\Sigma}_{i}<0$ in (2.39) is equivalent to $\Sigma_{i}<0$ in (2.45). From $\Sigma_{i}<0$ and by the similar derivation of Theorem 2.5, the proof can be completed.

If $D=0$, Theorem 2.11 can be reduced to the following corollary.

Corollary 2.12. Assume that for $\alpha>0,0 \leq \alpha_{i} \leq 1, i=1,2, \ldots, N$, there exist constants $\varepsilon_{i}>0$, $i=1,2, \ldots, N$, some $n \times n$ matrices $P, Q, R_{3}, R_{4}>0$, some $n \times n$ matrices $U, \widehat{S}_{i}, V_{1 i}, V_{2 i}$, and $V_{3 i}$, $i=1,2, \ldots, N$, such that the following $L M I$ conditions hold for all $i=1,2, \ldots, N$ :

$$
\begin{gathered}
\widehat{\Sigma}_{i}=\left[\begin{array}{cccccc}
\widehat{\Sigma}_{11 i} & \widehat{\Sigma}_{12 i} & \widehat{\Sigma}_{13 i} & \widehat{\Sigma}_{14 i} & \widehat{\Sigma}_{15 i} & \widehat{\Sigma}_{16 i} \\
* & \widehat{\Sigma}_{22 i} & \widehat{\Sigma}_{23 i} & 0 & \widehat{\Sigma}_{25 i} & 0 \\
* & * & \widehat{\Sigma}_{33 i} & \widehat{\Sigma}_{34 i} & 0 & \widehat{\Sigma}_{36 i} \\
* & * & * & \widehat{\Sigma}_{44 i} & 0 & 0 \\
* & * & * & * & \widehat{\Sigma}_{55 i} & 0 \\
* & * & * & * & * & \widehat{\Sigma}_{66 i}
\end{array}\right]<0, \\
F^{T} P+P F+\sum_{i=1}^{N} \alpha_{i} \cdot\left(\widehat{S}_{i}+\widehat{S}_{i}^{T}\right)+Q<0, \quad F=\sum_{i=1}^{N} \alpha_{i} A_{0 i},
\end{gathered}
$$


where

$$
\begin{gathered}
\widehat{\Sigma}_{11 i}=2 \alpha \cdot P-Q+R_{3}-V_{1 i}-V_{1 i}^{T}, \quad \widehat{\Sigma}_{12 i}=A_{0 i}^{T} U^{T}, \quad \widehat{\Sigma}_{13 i}=P A_{1 i}-\widehat{S}_{i}-V_{2 i}+V_{1 i}^{T}, \\
\widehat{\Sigma}_{14 i}=-\widehat{S}_{i}-V_{3 i}+V_{1 i}^{T}, \quad \widehat{\Sigma}_{15 i}=P M_{i}, \quad \widehat{\Sigma}_{16 i}=\varepsilon_{i} \cdot N_{0 i}^{T}, \quad \widehat{\Sigma}_{22 i}=h_{M}^{2} \cdot R_{4}-U-U^{T}, \\
\widehat{\Sigma}_{23 i}=U A_{1 i}, \quad \widehat{\Sigma}_{25 i}=U M_{i}, \quad \widehat{\Sigma}_{33 i}=-\left(1-h_{D}\right) \cdot e^{-2 \alpha h_{M}} \cdot R_{3}+V_{2 i}+V_{2 i}^{T}, \quad \widehat{\Sigma}_{34 i}=V_{3 i}+V_{2 i}^{T}, \\
\widehat{\Sigma}_{36 i}=\varepsilon_{i} \cdot N_{1 i}^{T}, \quad \widehat{\Sigma}_{44 i}=-e^{-2 \alpha h_{M}} \cdot R_{4}+V_{3 i}+V_{3 i}^{T}, \quad \widehat{\Sigma}_{55 i}=\widehat{\Sigma}_{66 i}=-\varepsilon_{i} \cdot I .
\end{gathered}
$$

Then the system (2.22a)-(2.22c) with $D=0$ is globally exponentially stabilizable with convergence rate $\alpha$ by the switching signal given in (2.37).

If $D=M_{i}=N_{0 i}=N_{1 i}=0, i=1,2, \ldots, N$, Corollary 2.12 can be reduced to the following corollary.

Corollary 2.13. Assume that for $\alpha>0,0 \leq \alpha_{i} \leq 1, i=1,2, \ldots, N$, there exist some $n \times n$ matrices $P, Q, R_{3}, R_{4}>0$, some $n \times n$ matrices $U, \widehat{S}_{i}, V_{1 i}, V_{2 i}$, and $V_{3 i}, i=1,2, \ldots, N$, such that the following LMI conditions hold for all $i=1,2, \ldots, N$ :

$$
\begin{gathered}
\widehat{\Sigma}_{i}=\left[\begin{array}{cccc}
\widehat{\Sigma}_{11 i} & \widehat{\Sigma}_{12 i} & \widehat{\Sigma}_{13 i} & \widehat{\Sigma}_{14 i} \\
* & \widehat{\Sigma}_{22 i} & \widehat{\Sigma}_{23 i} & 0 \\
* & * & \widehat{\Sigma}_{33 i} & \widehat{\Sigma}_{34 i} \\
* & * & * & \widehat{\Sigma}_{44 i}
\end{array}\right]<0, \\
F^{T} P+P F+\sum_{i=1}^{N} \alpha_{i} \cdot\left(\widehat{S}_{i}+\widehat{S}_{i}^{T}\right)+Q<0, \quad F=\sum_{i=1}^{N} \alpha_{i} A_{0 i},
\end{gathered}
$$

where $\widehat{\Sigma}_{j k i}, j, k=1,2,3,4, i=1,2, \ldots, N$, are defined in Corollary 2.12. Then the system (2.22a)(2.22c) with $D=M_{i}=N_{0 i}=N_{1 i}=0$ is globally exponentially stabilizable with convergence rate $\alpha$ by the switching signal given in (2.37).

Remark 2.14. By setting $\alpha=0$ in Theorems 2.5-2.11 and Corollaries 2.7-2.13, the global asymptotic stability for system (2.22a)-(2.22c) can be guaranteed.

\section{Numerical Examples}

Example 3.1. Consider the system (2.22a)-(2.22c) and the following parameters:

$$
N=2, \quad D=\left[\begin{array}{cc}
0.1 & 0 \\
0.1 & -0.1
\end{array}\right], \quad A_{01}=\left[\begin{array}{cc}
-4 & 2 \\
1 & 0
\end{array}\right], \quad A_{11}=\left[\begin{array}{cc}
1 & 0 \\
0.5 & 1
\end{array}\right], \quad A_{02}=\left[\begin{array}{cc}
0 & -1 \\
-1 & -4
\end{array}\right],
$$


Table 1: Comparison with other previous results.

Allowable time-varying delay bounds retaining global asymptotic stability $(\alpha=0)$ of the system (2.22a) $-(2.22 \mathrm{c})$ with (3.1) under switching signal (3.4)

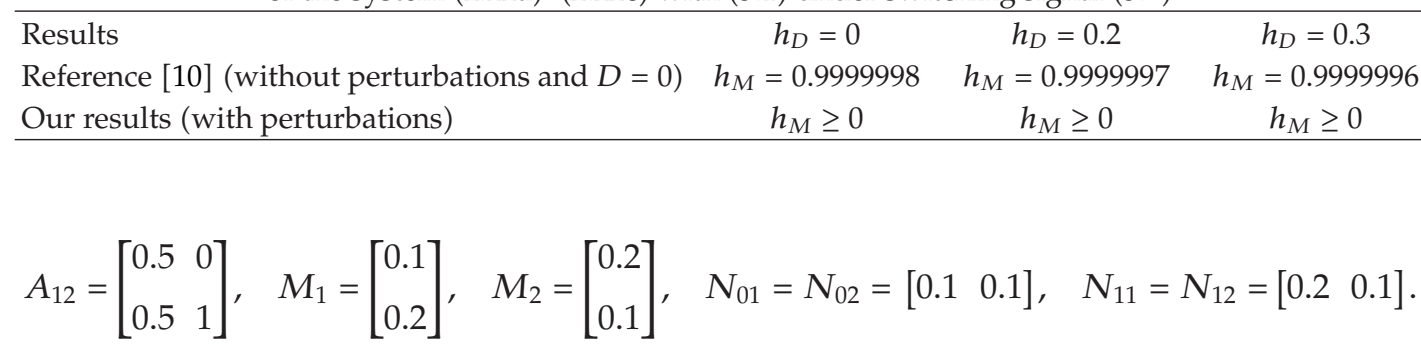

By Corollary 2.7, a feasible solution of LMI (2.29) with (3.1), $\alpha=0, h_{D}=0.2$, and $\alpha_{1}=\alpha_{2}=0.5$ is given by

$$
\begin{gathered}
P=\left[\begin{array}{cc}
169.0808 & 6.9691 \\
6.9691 & 147.4925
\end{array}\right], \quad Q=\left[\begin{array}{cc}
618.4486 & -21.7634 \\
-21.7634 & 538.3595
\end{array}\right], \quad R_{1}=\left[\begin{array}{cc}
120.4512 & -58.0284 \\
-58.0284 & 90.4631
\end{array}\right], \\
R_{2}=\left[\begin{array}{cc}
275.0262 & 25.6702 \\
25.6702 & 180.9190
\end{array}\right], \quad \varepsilon_{1}=145.7753, \quad \varepsilon_{2}=180.2272 .
\end{gathered}
$$

Select the switching signal by

$$
\sigma= \begin{cases}1, & x \in \bar{\Omega}_{1}, \\ 2, & x \in \bar{\Omega}_{2},\end{cases}
$$

where $\bar{\Omega}_{1}=\Omega_{1}, \bar{\Omega}_{2}=\Omega_{2}-\bar{\Omega}_{1}$,

$$
\begin{aligned}
& \Omega_{1}=\left\{x \in \mathfrak{R}^{n}:-720.2596 x_{1}^{2}+872.0285 x_{1} x_{2}+566.2359 x_{2}^{2} \leq 0\right\}, \\
& \Omega_{2}=\left\{x \in \Re^{n}: 604.5104 x_{1}^{2}-732.4262 x_{1} x_{2}-655.5187 x_{2}^{2} \leq 0\right\} .
\end{aligned}
$$

The switching regions $\Omega_{1}$ and $\Omega_{2}$ are sketched in Figure 1. The system (2.22a)-(2.22c) with $h_{D}=0.2$ and (3.1) is globally asymptotically stabilizable by the switching signal (3.4). Some comparisons are made in Table 1. The result of this paper provides a major improvement to guarantee the global asymptotic stability of system (2.22a)-(2.22c) with (3.1). 


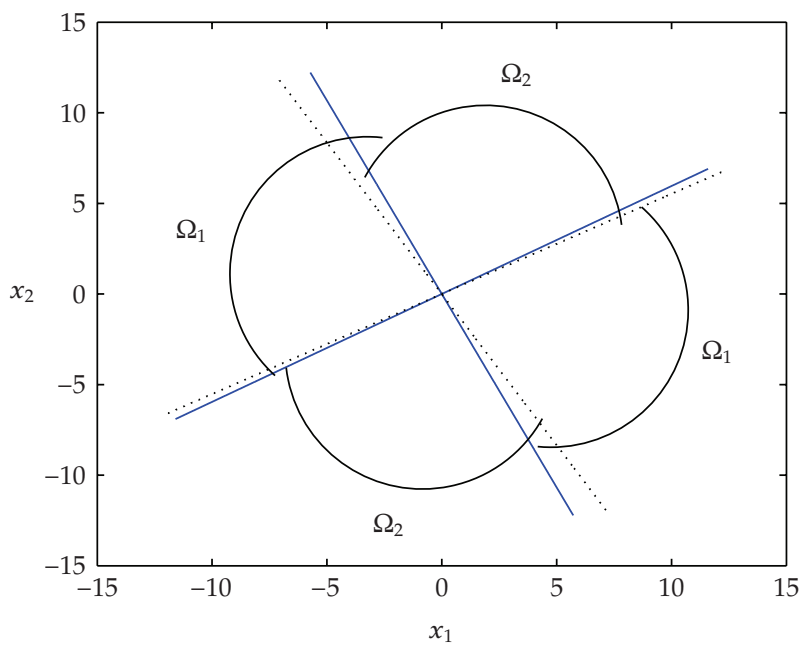

Figure 1: Switching regions.

Table 2: Comparison with other previous results.

\begin{tabular}{cccccc}
\hline \multicolumn{5}{c}{ Allowable time-varying delay bounds retaining global asymptotic stability $(\alpha=0)$} \\
of the system $(2.22 \mathrm{a})-(2.22 \mathrm{c})$ with $(3.6)$ \\
\hline Results & $h_{D}=0$ & $h_{D}=0.1$ & $h_{D}=0.5$ & $h_{D}=0.9$ & $h_{D}=1$ \\
\hline Reference [7] & $h_{M}=0.001573$ & \multicolumn{5}{c}{ No results } \\
\hline Reference [6] & $h_{M}=0.00392$ & \multicolumn{4}{c}{ No results } \\
\hline Reference [10] & $h_{M}=0.0202$ & $h_{M}=0.0179$ & $h_{M}=0.0176$ & $h_{M}=0.0176$ & $h_{M}=0.0176$ \\
Our results & $h_{M}=0.0309$ & $h_{M}=0.0271$ & $h_{M}=0.0193$ & $h_{M}=0.0181$ & $h_{M}=0.0180$ \\
\hline
\end{tabular}

Example 3.2. Consider the system (2.22a)-(2.22c) and the following parameters [7]:

$$
\begin{array}{ll}
N=2, \quad D=M_{i}=N_{0 i}=N_{1 i}=0, & A_{01}=\left[\begin{array}{cc}
-2 & 2 \\
-20 & -2
\end{array}\right], \\
A_{11}=\left[\begin{array}{cc}
-1 & -7 \\
23 & 6
\end{array}\right], \quad A_{02}=\left[\begin{array}{ll}
-2 & 10 \\
-4 & -2
\end{array}\right], & A_{12}=\left[\begin{array}{ll}
4 & -5 \\
1 & -8
\end{array}\right] .
\end{array}
$$

By Corollary 2.13, some comparisons with the obtained results for switched system (2.22a)-(2.22c) with (3.6) are made in Table 2. The results of this paper provide a larger allowable upper bound for time delay to guarantee the global asymptotic stability of system (2.22a) $-(2.22 \mathrm{c})$ with (3.6) by the switching signal (2.37).

Example 3.3. Consider the following switched system with input time delay [7]:

$$
\dot{x}(t)=A_{0 \sigma} x(t)+B_{\sigma} u(t-h(t)), \quad t \geq 0,
$$


Table 3: Comparison with other previous results.

Allowable time-varying delay bounds retaining global asymptotic and exponential stability of the system (3.7) with (3.9)

\begin{tabular}{ccccc}
\hline Results & $h_{D}=0, \alpha=0$ & $h_{D}=0.1, \alpha=0$ & $h_{D}=0, \alpha=0.1$ & $h_{D}=0, \alpha=0.2$ \\
\hline Reference [7] & $h_{M}=0.008723$ & \multicolumn{3}{c}{ No results } \\
\hline Reference [10] & $h_{M}=0.0189$ & $h_{M}=0.0182$ & \multicolumn{2}{c}{ No results } \\
\hline Our results & $h_{M}=0.03112$ & $h_{M}=0.0294$ & $h_{M}=0.0296$ & $h_{M}=0.0281$ \\
\hline
\end{tabular}

where

$$
N=2, \quad A_{01}=\left[\begin{array}{cc}
3 & 2 \\
-5 & -1
\end{array}\right], \quad A_{02}=\left[\begin{array}{cc}
-1 & 20 \\
-2 & 2
\end{array}\right], \quad B_{1}=\left[\begin{array}{cc}
-1 & 0 \\
1 & -1
\end{array}\right], \quad B_{2}=\left[\begin{array}{cc}
1 & 1 \\
0 & -1
\end{array}\right] .
$$

The feedback control is given by $u(t)=K_{\sigma(t)} x(t)$ with

$$
K_{1}=\left[\begin{array}{cc}
5 & 0 \\
20 & 1
\end{array}\right], \quad K_{2}=\left[\begin{array}{cc}
-3 & -14 \\
2 & 4
\end{array}\right] .
$$

For the given feedback control (3.9), system (3.7) can be rewritten as

$$
\dot{x}(t)=A_{0 \sigma} x(t)+A_{1 \sigma} x(t-h(t)), \quad t \geq 0,
$$

where $A_{1 \sigma}=B_{\sigma} K_{\sigma}$. As shown in Table 3, the results obtained in this paper provide larger allowable time delay bounds guaranteeing the global stability of system (3.7) with (3.9) by switching signal $(2.37)$. In $[7,10]$, the convex combination parameters are chosen by $\alpha_{1}=1 / 3$ and $\alpha_{2}=2 / 3$. The convex combination parameters of our results are chosen by $\alpha_{1}=0.1$ and $\alpha_{2}=0.9$.

\section{Conclusions}

In this paper, the switching signal design for global exponential stability of uncertain switched neutral systems with mixed time delays has been considered. LMI and Razumikhinlike approaches are used to derive delay-dependent and delay-independent stability criteria. The results obtained in this paper are less conservative than the previous ones for the numerical examples investigated in this paper.

\section{Acknowledgment}

The research reported here was supported by the National Science Council of Taiwan, under Grant no. NSC 96-2221-E-022-011-MY2. 


\section{References}

[1] K. Gu, V. L. Kharitonov, and J. Chen, Stability of Time-Delay Systems, Birkhäuser, Boston, Mass, USA, 2003.

[2] J. K. Hale and S. M. Verduyn Lunel, Introduction to Functional-Differential Equations, vol. 99 of Applied Mathematical Sciences, Springer, New York, NY, USA, 1993.

[3] V. B. Kolmanovskii and A. Myshkis, Applied Theory of Functional Differential Equations, Kluwer Academic Publishers, Dordrecht, The Netherlands, 1992.

[4] C.-H. Lien and K.-W. Yu, "Non-fragile $H_{\infty}$ control for uncertain neutral systems with time-varying delays via the LMI optimization approach," IEEE Transactions on Systems, Man, and Cybernetics, Part $B$, vol. 37, no. 2, pp. 493-499, 2007.

[5] D. Xie, N. Xu, and X. Chen, "Stabilisability and observer-based switched control design for switched linear systems," IET Control Theory E Applications, vol. 2, no. 3, pp. 192-199, 2008.

[6] R. Chen and K. Khorasani, "Stability analysis of a class of switched time-delay systems with unstable subsystems," in Proceedings of the IEEE International Conference on Control and Automation (ICCA '07), pp. 265-270, GuangZhou, China, May-June 2007.

[7] S. Kim, S. A. Campbell, and X. Liu, "Stability of a class of linear switching systems with time delay," IEEE Transactions on Circuits and Systems I, vol. 53, no. 2, pp. 384-393, 2006.

[8] V. Kulkarni, M. Jun, and J. Hespanha, "Piecewise quadratic Lyapunov functions for piecewise affine time-delay systems," in Proceedings of the American Control Conference (AAC '04), vol. 5, pp. 3885-3889, Boston, Mass, USA, June-July 2004.

[9] J. Liu, X. Liu, and W.-C. Xie, "Delay-dependent robust control for uncertain switched systems with time-delay," Nonlinear Analysis: Hybrid Systems, vol. 2, no. 1, pp. 81-95, 2008.

[10] X.-M. Sun, W. Wang, G.-P. Liu, and J. Zhao, "Stability analysis for linear switched systems with timevarying delay," IEEE Transactions on Systems, Man, and Cybernetics, Part B, vol. 38, no. 2, pp. 528-533, 2008.

[11] Y. G. Sun, L. Wang, and G. Xie, "Stability of switched systems with time-varying delays: delaydependent common Lyapunov functional approach," in Proceedings of the American Control Conference, vol. 5, pp. 1544-1549, Minneapolis, Minn, USA, June 2006.

[12] X.-M. Sun, J. Zhao, and D. J. Hill, "Stability and $L_{2}$-gain analysis for switched delay systems: a delaydependent method," Automatica, vol. 42, no. 10, pp. 1769-1774, 2006.

[13] C.-H. Wang, L. I.-X. Zhang, H.-J. Gao, and L.-G. Wu, "Delay-dependent stability and stabilization of a class of linear switched time-varying delay systems," in Proceedings of the 4th International Conference on Machine Learning and Cybernetics (ICMLC'05), pp. 18-21, GuangZhou, China, 2005.

[14] C.-H. Lien, K.-W. Yu, Y.-F. Lin, Y.-J. Chung, and L.-Y. Chung, "Exponential convergence rate estimation for uncertain delayed neural networks of neutral type," Chaos, Solitons and Fractals, vol. 40, no. 5, pp. 2491-2499, 2009.

[15] S. Boyd, L. El Ghaoui, E. Feron, and V. Balakrishnan, Linear Matrix Inequalities in System and Control Theory, vol. 15 of SIAM Studies in Applied Mathematics, SIAM, Philadelphia, Pa, USA, 1994. 


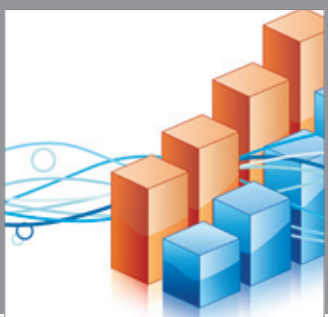

Advances in

Operations Research

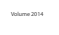

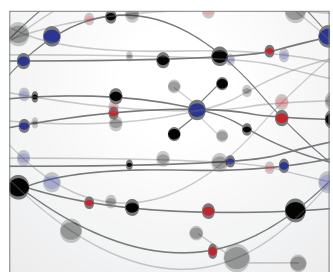

\section{The Scientific} World Journal
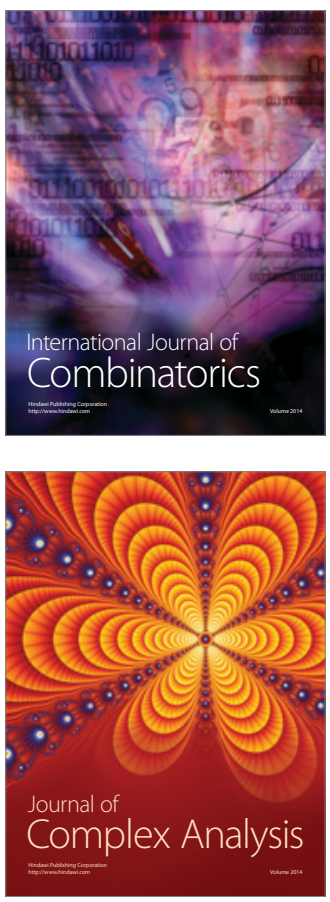

International Journal of

Mathematics and

Mathematical

Sciences
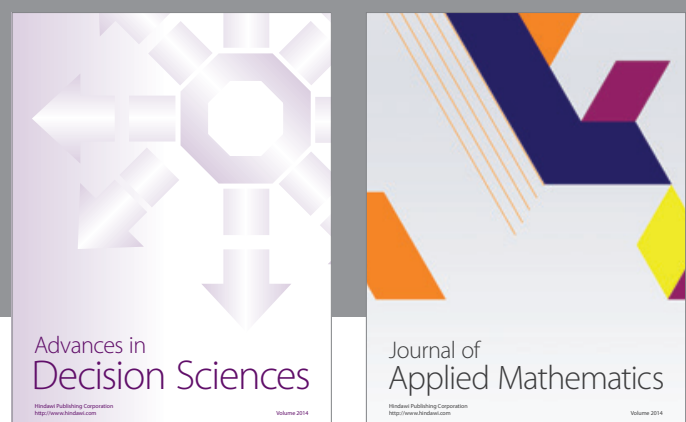

Journal of

Applied Mathematics
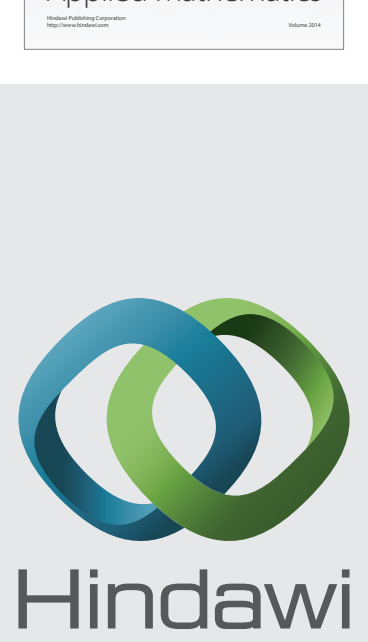

Submit your manuscripts at http://www.hindawi.com
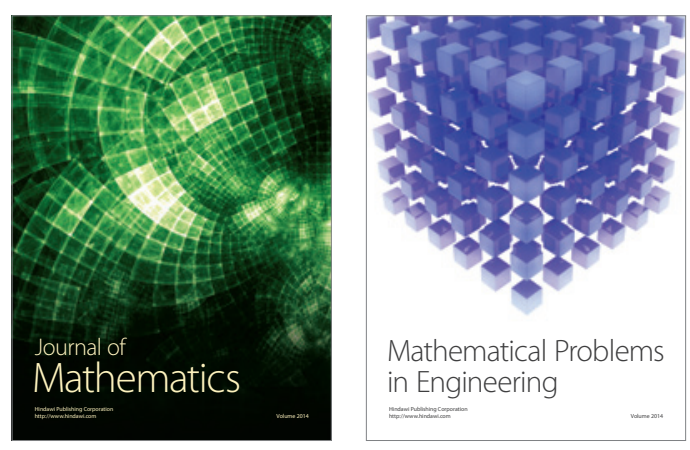

Mathematical Problems in Engineering
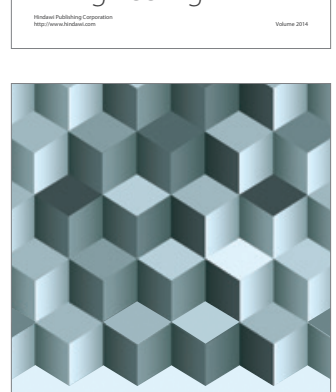

Journal of

Function Spaces
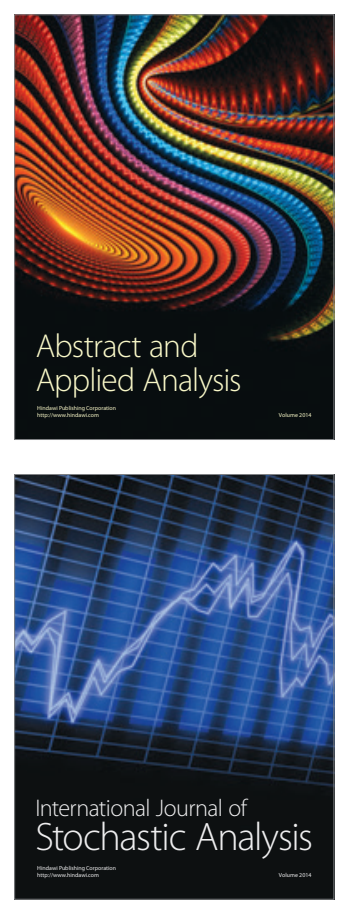

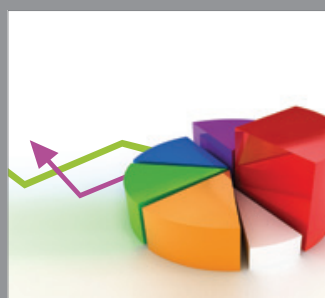

ournal of

Probability and Statistics

Promensencen
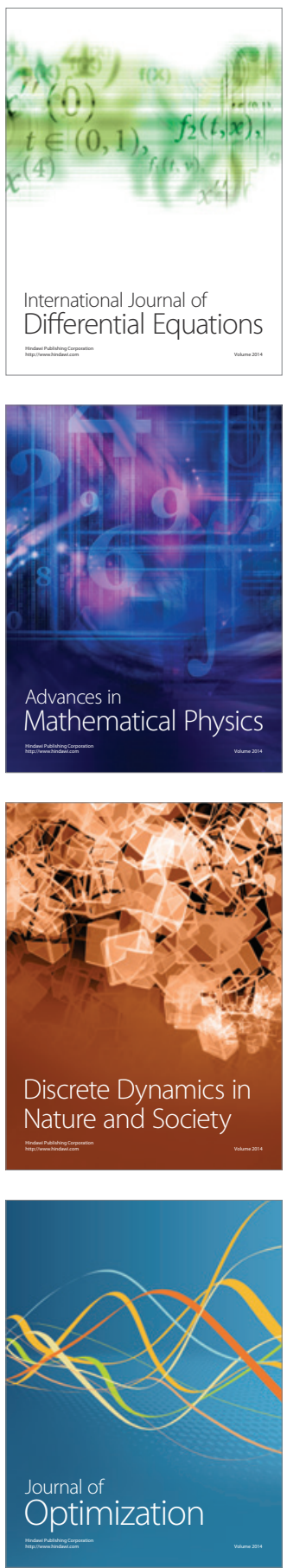